\title{
Primary pseudomyogenic hemangioendothelioma of the vulva: a rare location for a rare entity
}

\author{
Yue-Fang Sun ${ }^{1 *}$ and Jian Wang ${ }^{2}$
}

\begin{abstract}
Background: Pseudomyogenic hemangioendothelioma (PMHE) is a recently described vascular neoplasm which typically occurs in the lower extremities of young to middle-aged adults.

Case presentation: We present here a unique case of PMHE arising primarily in the vulva of a 51-year-old woman who presented with a painful vulvar nodule. Clinically, it was thought as Bartholin gland cyst, vulvar hematoma or papilloma. On surgery, two nodules were found with one located in the superficial dermis and the other in the deep subcutis. Histologically, these two nodules showed similar features, composed of fascicles or sheets of plump spindled to epithelioid cells with eosinophilic cytoplasm. Given the morphological resemblance to a myogenic tumor, the lesion was initially diagnosed as a rhabdomyosarcoma by the referring pathologist. However, a comprehensive reevaluation of the submitted slides made us reconsider a PMHE, which was subsequently confirmed by immunohistochemical study.

Conclusion: This case demonstrates that PMHE can also develop in the female external genitalia albeit extremely rare. This disease should be included in the differential diagnostic list of vulvar tumors with spindled to epithelioid morphology and cytokeratin-positive immunophenotypes.
\end{abstract}

Keywords: Vulva, Pseudomyogenic hemangioendothelioma, Immunohistochemistry

\section{Background}

Pseudomyogenic hemangioendothelioma (PMHE), also known as epithelioid sarcoma-like hemangioendothelioma [1], is a rare vascular neoplasm of biologically intermediate malignancy. This rare tumor type typically occurs in the lower extremities of young to middle-aged adults with a striking male predominance [2]. Less commonly, it may involve upper extremities, trunk, pelvis, and head and neck region [3, 4]. Multicentric synchronous presentation with involvement of different tissue planes in an anatomic region is a common feature of PMHE. Due to the lack of morphological evidence of vasoformation, a vascular neoplasm is hardly considered by pathologists especially for those who lack expertise in soft tissue tumors. On the contrary, because of the

\footnotetext{
* Correspondence: arthur180134@126.com

1Department of Pathology, Shanghai Tenth People's Hospital, Tenth People's Hospital of Tongji University, 301 Yanchang Middle Road, Shanghai 200072, China

Full list of author information is available at the end of the article
}

cytomorphological striking resemblance to rhabdomyoblasts, it is not uncommon to misdiagnose PMHE as a myogenic tumor. In this report, we present such a case of PMHE that arose in the vulva of a middle-age woman which was initially thought as a rhabdomyosarcoma. To the best of our knowledge, this is the first case of PMHE arising primarily in the female external genitalia.

\section{Case presentation}

The patient was a 51-year-old woman (gravida 2, para 2) who felt itchiness in the right vulva for 3 months. She scratched at whiles and recently noted a small painful nodule in the right vulva. She went to a clinic for medical attention. She was given external medicine for alleviation but turned to be less effective. She was then admitted to a local hospital for further treatment. Gynecological examination revealed two pea-sized solid nodules with tenderness affecting the right labium majus. Clinically, they were suspected as Bartholin gland cysts, vulvar hematomas or papillomas. On surgery, one

(C) The Author(s). 2019 Open Access This article is distributed under the terms of the Creative Commons Attribution 4.0 International License (http://creativecommons.org/licenses/by/4.0/), which permits unrestricted use, distribution, and 
nodule was found to be located in the superficial dermis and the other in the deep subcutis, both measuring approximately $1 \mathrm{~cm}$ in maximum diameter. Considering benign lesion, marginal resection was performed. It was interpreted pathologically as a rhabdomyosarcoma, with proliferative fasciitis needed to be excluded. In view of potential further treatment, the pathological materials were sent to us for further confirmation. After the final diagnosis of PMHE was rendered, the patient was recommended to have a thorough radiological examination, including PET-CT. There was no neoplastic disease elsewhere. A three-month-follow-up showed no signs of local recurrence or metastatic disease.

Grossly, one specimen tagged "mass of right labium majus (epidermis)" consisted of a $1.5 \times 1 \times 1 \mathrm{~cm}$ fibroadipose tissue covered with a $1.5 \times 1 \mathrm{~cm}$ elliptical skin. On cut section, there was a solid gray nodule, measuring $1.5 \times 0.8 \times 0.5 \mathrm{~cm}$ in size and was intermediate to firm in consistency. The other specimen tagged "mass of right labium majus (deep)" consisted mainly of adipose tissue, measuring $2 \times 1 \times 0.8 \mathrm{~cm}$ in total volume. On cut section, there was presence of solid grayish area, measuring about $1 \mathrm{~cm}$ in maximum size.

Microscopically, the "mass of right labium majus (epidermis)" was dermal-based (Fig. 1a, b), whereas the "mass of right labium majus (deep)" was located within the subcutaneous adipose tissue, assuming multinodular architecture (Fig. 1c). On high power, they were composed of fascicles or sheets of plump spindled to epithelioid cells with abundant eosinophilic cytoplasm, closely resembling rhabdomyoblasts (Figs. 1d, e). Focal storiform arrangement was also present (Figs. 1f). The tumor cells had oval to round vesicular nuclei with small nucleoli. Nuclear atypia is mild and mitotic activity was scarce $(<5 / 50$ high power field). There was a prominent neutrophilic infiltration in the stroma. Coagulative necrosis was absent.

Immunochemically, tumor cells were diffusely positive for AE1/AE3, ERG and Fli1 (Fig. 2a, b), and partially positive for CD31 (Fig. 2c). The Ki67 index was about 2\% (Fig. 2d). Intact staining of INI-1(SMARCB1) was retained. The tumor cells were negative for EMA, CD34, $\alpha$-SMA, MSA, desmin, myogenin, MyoD1, calponin, ER
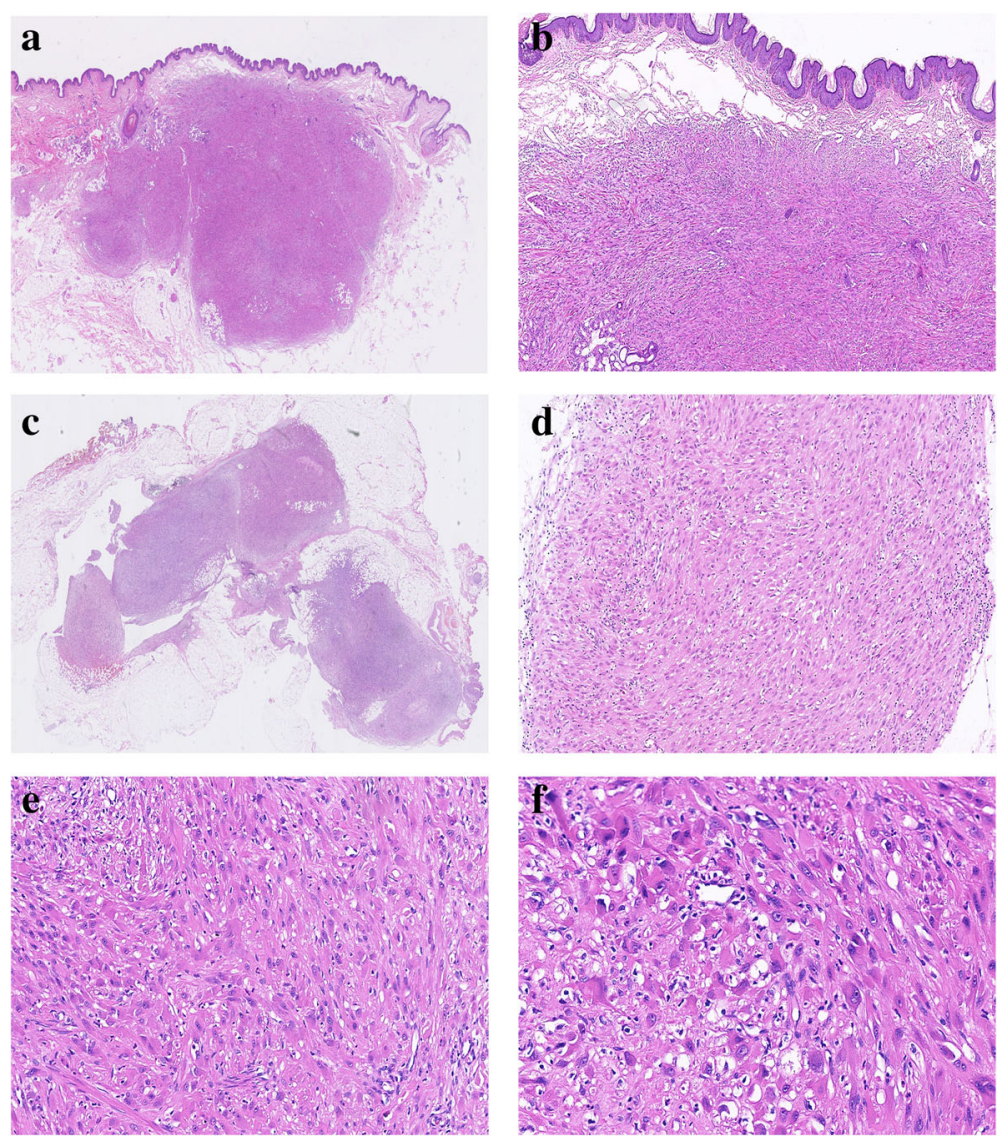

Fig. 1 Scanning image of "mass of right labium majus (epidermis)" displayed a vague nodular appearance of the tumor (a), which was mainly dermal-based (b). Scanning image of "mass of right labium majus (deep)" showed a subcutaneously located tumor with multinodular architecture (c). The tumor was composed of fascicles of plump spindled to epithelioid cells with abundant eosinophilic cytoplasm (d), some of which resembled rhabdomyoblasts (e). Focal storiform arrangement was present and scattered neutrophilic infiltrate was observed in the stroma (f). 

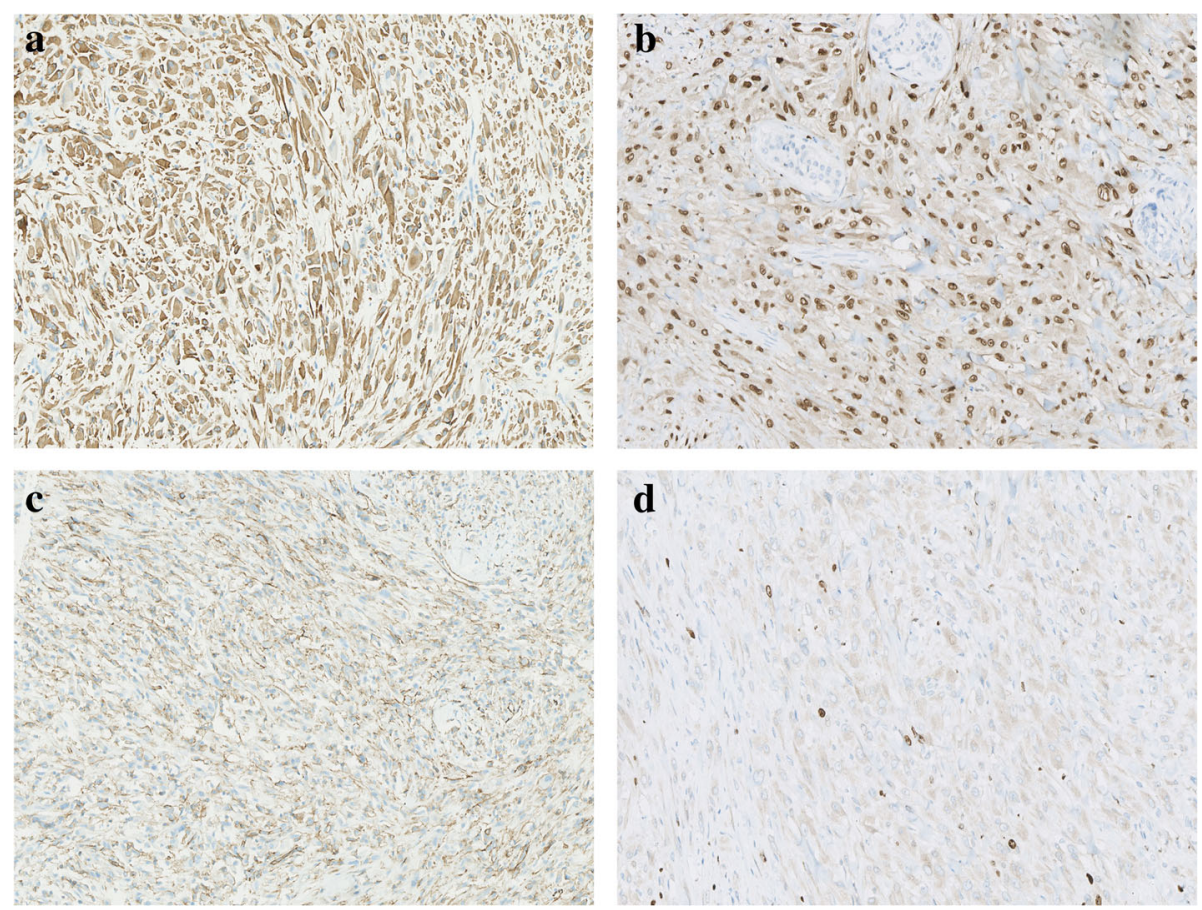

Fig. 2 Tumor cells showed diffuse staining of AE1/AE3 (a) and ERG (b) with partial expression of CD31 (c). The Ki67 index was relatively low (d).

and PR. The results of immunochemical study were summarized in Table 1.

\section{Discussion and conclusion}

The morphology and immunophenotype of the current case are in consistency with a typical PMHE, a

Table 1 Immunohistochemical results of pseudomyogenic hemangioendothelioma

\begin{tabular}{lllll}
\hline Antibody & Clone & Source & Dilution & Result \\
\hline Pancytokeratin & AE1/AE3 & Dako & $1: 100$ & + \\
EMA & E29 & Dako & $1: 200$ & - \\
CD31 & JC70A & Dako & $1: 80$ & + \\
CD34 & QBend 10 & Dako & $1: 50$ & - \\
ERG & EPR3864 & Roche & Ready-to-use & + \\
Fli1 & G146-222 & BD Biosciences & $1: 100$ & + \\
a-SMA & 1A4 & Dako & $1: 400$ & - \\
MSA & HHF35 & Dako & $1: 300$ & - \\
Desmin & D33 & Dako & $1: 500$ & - \\
Calponin & CALP & Maixin China & $1: 150$ & - \\
Myogenin & MYF4 & Novocastra & $1: 500$ & - \\
MyoD1 & MYF3 & Dako & $1: 50$ & - \\
SMARCB1(IN11) & 25/BAF47 & BD Biosciences & $1: 50$ & Retained \\
ER & PPG5/10 & Dako & $1: 35$ & - \\
PR & PgR 636 & Dako & $1: 50$ & - \\
Ki67 & MIB1 & Dako & $1: 150$ & $2 \%$ \\
\hline
\end{tabular}

distinctive vascular neoplasm which has been included in the 4th edition of WHO classification of Soft Tissue and Bone Tumors [5]. Although being well recognized as a novel entity of hemangioendothelioma, PMHE is known for lack of morphological evidence of endothelial differentiation. The descriptive term of 'pseudomyogenic' attributes to the neoplastic cells' striking resemblance to rhabdomyoblasts in cytomorphology, which may lead to a misdiagnosis of a myogenic neoplasm, as illustrated in the current case as well as reported in the literature [6].

PMHE represents a rare neoplasm. Up to present, approximately 140 cases of PMHE have been reported in the English literature [7-9]. There was a male predominance with a gender ratio of 3.3:1. The majority occurred in young to middle aged adults with a mean and median age of 32 and 27 years respectively (range: 5 to 82 years). The peak age of incidence was in the second to fourth decades, which accounted for about $70 \%$ of all patients. Children (younger than 10 years) and elderly people (older than 60 years) were rarely involved.

Approximately $75 \%$ of PMHE arose in the skin and soft tissues, including those with concurrence of bone. Nearly one fourth of cases presented as primary bone lesions $[10,11]$. With regard to site, over half of PMHE occurred in the lower extremities, including foot, leg, knee and thigh. About $17 \%$ occurred in the upper extremities, including forearm, hand, finger, upper arm, wrist and axilla; $15 \%$ in the trunk, including abdominal 
wall, chest wall, back and pelvis; $6 \%$ in the head and neck region, including face, oral cavity and neck; and exceedingly unusual site like the penis [12]. To date, occurrence of PMHE in the vulva has not been reported thus far. The current case is the first case of PMHE arising in the female external genitalia.

The clinical manifestation of PMHE was nonspecific. Most patients presented with multiple small painless or painful nodules which were rarely suspected as vascular lesions by clinicians. The clinical diagnosis embraced a wide variety of disease, including cutaneous cyst, sebaceous cyst, mosquito bite, abscess, nodular fasciitis, keratoacanthoma, spiradenoma, dermatofibroma, leiomyoma, neuroma, hemangioma and angiosarcoma [2, 7, 12-15]. In our case, the lesion was originally considered Bartholin gland cyst, vulvar hematoma or papilloma. It is worthy to note that patients with PMHE may present with a few visible cutaneous nodules which might not arise the clinicians' alert. As about 70\% of PMHE presented with multifocal disease which may involve multiple tissue planes in an anatomic region, it is essential for the patients to have further radiological examinations to make sure if there are occult intramuscular nodules or associated concurrence of bone lesions $[2,16]$.

On histological examination, our case displayed the characteristic features of PMHE that consisted of fascicles and sheets of plump spindle-to round shaped cells with vesicular nuclei, inconspicuous nucleoli and abundant homogeneous eosinophilic cytoplasm, mimicking rhabdomyoblasts. However, neoplastic cells were negative for myogenic markers such as desmin and myogenin. The epithelioid cytomorphology and immunoreactivity of AE1/ AE3 might suggest an epithelioid sarcoma, one of SWI/ SNF complex-deficient soft tissue neoplasms, which may also occur in the vulvar region. However, EMA and CD34 immunonegativity, and intact expression of INI1 in PMHE helped in the distinction from epithelioid sarcoma. Because of epithelioid morphology and endothelial differentiation, PMHE need to be differentiated from epithelioid vascular neoplasms, including epithelioid hemangioendothelioma, epithelioid hemangioma and epithelioid angiosarcoma. Epithelioid hemangioendothelioma (EHE) is characterized by cords and nests of epithelioid cells in a myxohyaline stroma. Tumor cells in EHE with WWTR1CAMTA1 fusion typically express CAMTA1 or show CAMTA1 gene rearrangement by molecular analysis. A small percentage of EHE with YAP1-TFE3 fusion express TFE3 or harbor TFE3 gene rearrangement [17]. Epithelioid hemangioma is a benign vascular neoplasm composed of well-formed vessels lined by epithelioid endothelial cells with abundant eosinophilic cytoplasm and enlarged round nuclei. In contrast, PMHE lacks well-formed neoplastic vessels. Epithelioid angiosarcoma is a malignant vascular tumor composed of sheets of large, atypical epithelioid cells with vesicular nuclei containing prominent large central nucleoli, often showing focal vasoformation [18].

The endothelial nature of PMHE was usually demonstrated with the application of a panel of endothelial markers, including CD31, ERG and Fli1. Whereas ERG was consistently expressed in PMHE, the expression of CD31 was only seen in about 50 60\% of PMHE. FOSB, a new marker derived from SERPINE1-FOSB fusion transcripts, was considered a highly sensitive in PMHE as diffuse nuclear immunoreactivity for FOSB $(>50 \%$ of cells) has been shown greater than $96 \%[19,20]$. As diffuse nuclear staining of FOSB was rarely observed in histologic mimics, FOSB was considered a diagnostic adjunct. However, what needs to be pointed out is that diffuse FOSB staining could be also observed in $54 \%$ of epithelioid hemangioma, and occasional cases of proliferative fasciitis, nodular fasciitis, and rarely epithelioid angiosarcoma, spindle cell angiosarcoma and epithelioid hemangioendothelioma [20, 21]. In addition, focal expression of FOSB has been demonstrated in a variety of lesions, including $50 \%$ of epithelioid sarcoma, $40 \%$ of epithelioid angiosarcoma, 55\% of nodular fasciitis, and 40\% of cellular fibrous histiocytoma [20]. Therefore, focal weak staining of FOSB was thought not diagnostic.

At molecular level, a balanced t (7;19)(q22;q13) has been found as the sole anomaly in PMHE [22]. Subsequent studies validated SERPINE1-FOSB gene fusion as the recurrent genetic alteration in PMHE [23]. Recently, a novel $A C T B-F O S B$ gene fusion has been identified in PMHE by means of MSK-fusion solid assay [24]. It was found that tumors harboring $A C T B-F O S B$ fusion more often presented with a solid pattern compared to those associated with SERPINEI-FOSB fusion [3]. FISH has been increasingly used in routine practice in the genetic analysis of translocation-related tumors. A precise diagnosis of PMHE can be also reached by using FISH analysis with split apart probes flanking SERPINE1/ACTB and FOSB genes, and further confirmed by RT-PCR assay [12].

Biologically, most of PMHE showed an indolent clinical course with a predilection for local recurrence, the rate of which was about $20 \%$ after a period ranging from 2 to 96 months after diagnosis [1, 2, 8]. Approximately one tenth of patients developed metastatic disease, including lung, regional lymph nodes, groin, subpleural region, ribs, and vertebrae [2, 11, 13, 25-29]. Few reports demonstrated that a PMHE could have a rapid progression and aggressive behavior [16, 30]. To date, only one case with numerous lytic bone lesions showed a spontaneous regression of the disease with PET/CT [27]. To avoid any risk of relapse, our patient had undergone a supplementary wide local excision and she remained well with no evidence of disease at last follow-up.

In the context of treatment, local resection is the mainstay for patients presenting with a localized disease. 
For patients with multifocal unresectable lesions or suffering metastatic disease, systemic chemotherapy is recommended. Most recently, target therapies such as mTOR inhibitors sirolimus, everolimus and rapamycin have been demonstrated to be effective treatment options in PMHE $[28,29]$. It was supposed that treatment with the mTOR inhibitors resulted in a reduction in expression of the SERPINE-FOSB fusion protein. Similar inhibitory effect has been shown with telatinib treatment [31], an available VEGFR1-4/PDGFRA inhibitor which blocked VEGF signaling and down-regulated SERPINE1, affecting the self-regulation of the fusion gene. Target therapy provided a promising treatment option for patients with inoperable PMHE that failed with standard traditional chemotherapy.

In summary, we present a unique case of PMHE which occurred primarily in the vulva of a 51-year-old woman. Albeit exceedingly rare, PMHE should be included in the differential diagnostic list of vulvar tumors with spindled to epithelioid morphology and cytokeratinpositive immunophenotypes.

\section{Abbreviations}

FISH: Fluorescenece in situ hybridization; PET/CT: Positron emission tomography/computed tomography; PMHE: Pseudomyogenic hemangioendothelioma

\section{Acknowledgements}

None.

\section{Authors' contributions}

Both authors performed the histological and immunohistochemical evaluation as well as collected clinical data and drafted the manuscript. Both authors read and approved the final manuscript.

\section{Funding}

No funding has been gained by the authors for this research.

\section{Availability of data and materials}

Please contact author for data requests.

\section{Ethics approval and consent to participate}

The ethical approval and documentation for a case report was waived with the Shanghai Tenth People's Hospital, Tenth People's Hospital of Tongji University.

\section{Consent for publication}

For this case report was retrieved from the consultant files, both authors cannot get in touch with the patient.

\section{Competing interests}

The authors declare that they have no competing interests.

\section{Author details}

'Department of Pathology, Shanghai Tenth People's Hospital, Tenth People's Hospital of Tongji University, 301 Yanchang Middle Road, Shanghai 200072, China. ${ }^{2}$ Department of Pathology, Fudan University Shanghai Cancer Center, Shanghai 200032, China.

Received: 5 April 2019 Accepted: 17 June 2019

Published online: 26 June 2019

\section{References}

1. Billings SD, Folpe AL, Weiss SW. Epithelioid sarcoma-like hemangioendothelioma. Am J Surg Pathol. 2003;27:48-57.
2. Hornick JL, Fletcher CDM. Pseudomyogenic hemangioendothelioma: a distinctive, often multicentric tumor with indolent behavior. Am J Surg Pathol. 2011;35:190-201.

3. Agaram NP, Zhang L, Cotzia P, Antonescu CR. Expanding the Spectrum of genetic alterations in Pseudomyogenic Hemangioendothelioma with recurrent novel ACTB-FOSB gene fusions. Am J Surg Pathol. 2018;42:1653-61.

4. Rawal YB, Anderson KM, Dodson TB. Pseudomyogenic Hemangioendothelioma: a vascular tumor previously undescribed in the Oral cavity. Head Neck Pathol. 2017;11:525-30.

5. Fletcher CDM, Bridge JA, Hogendoorn PCW, et al. WHO classification of Tumours of soft tissue and bone. Lyon: IARC Press; 2013.

6. Rekhi B, Gulia A, Rangarajan V. A rare case of multifocal pseudomyogenic hemangioendothelioma, involving soft tissues and bone, misdiagnosed as a rhabdomyosarcoma: diagnostic and treatment implications. Indian J Pathol Microbiol. 2016;59:382-5.

7. Raftopoulos E, Royer M, Warren M, Zhao J, Rush W. Pseudomyogenic Hemangioendothelioma: case report and review of the literature. Am J Dermatopathol. 2018;40:597-601.

8. Pradhan D, Schoedel K, McGough RL, Ranganathan S, Uma NMR. Pseudomyogenic hemangioendothelioma of skin, bone and soft tissue-a clinicopathological, immunohistochemical, and fluorescence in situ hybridization study. Hum Pathol. 2017;71:126-34 Elsevier Inc.

9. Santos RP, Carvalho S, Joana G, Perdal J. Pseudomyogenic hemangioendothelioma: a little-known tumor. Acta Dermatovenerol Alp Pannonica Adriat. 2018;27:225-9.

10. Sheng W, Wang J. Primary pseudomyogenic hemangioendothelioma of bone. Histopathology. 2012;61:1219-24.

11. Inyang A, Mertens F, Puls F, Sumathi V, Inwards C, Folpe A, et al. Primary pseudomyogenic hemangioendothelioma of bone. Am J Surg Pathol. 2016; 40:587-98.

12. Ide YH, Tsukamoto $Y$, Ito T, Watanabe T, Nakagawa N, Haneda T, et al. Penile pseudomyogenic hemangioendothelioma/epithelioid sarcoma-like hemangioendothelioma with a novel pattern of SERPINE1-FOSB fusion detected by RT-PCR - report of a case. Pathol Res Pract. 2015;211:415-20 Elsevier GmbH.

13. Amary MF, O'Donnell P, Berisha F, Tirabosco R, Briggs T, Pollock R, et al. Pseudomyogenic (epithelioid sarcoma-like) hemangioendothelioma: characterization of five cases. Skelet Radiol. 2013;42:947-57.

14. Cheo FF, Pseudomyogenic SK. (Epithelioid sarcoma-like) hemangioendothelioma - a rare vascular neoplasm with deceptive morphology and distinctive immunophenotype. Malays J Pathol. 2017;39:305-9.

15. Stuart LN, Gardner JM, Lauer SR, Monson DK, Parker DC, Edgar MA. Epithelioid sarcoma-like (pseudomyogenic) hemangioendothelioma, clinically mimicking dermatofibroma, diagnosed by skin biopsy in a 30-yearold man. J Cutan Pathol. 2013;40:909-13.

16. Sheng W, Pan Y, Wang J. Pseudomyogenic hemangioendothelioma: report of an additional case with aggressive clinical course. Am J Dermatopathol. 2013;35:597-600.

17. Flucke U, Vogels RJC, de Saint Aubain Somerhausen N, Creytens DH, Riedl RG, van Gorp JM, et al. Epithelioid Hemangioendothelioma: clinicopathologic, immunhistochemical, and molecular genetic analysis of 39 cases. Diagn Pathol. 2014;9:131-43.

18. Shon W, Billings SD. Epithelioid vascular tumors : a review. Adv Anat Pathol. 2019;00:1-12

19. Sugita S, Hirano H, Kikuchi N, Kubo T, Asanuma H, Aoyama T, et al. Diagnostic utility of FOSB immunohistochemistry in pseudomyogenic hemangioendothelioma and its histological mimics. Diagn Pathol. 2016:11:75-80.

20. Hung YP, Fletcher CDM, Hornick JL. FOSB is a useful diagnositic marker for pseudomyogenic hemangioendothelioma. Am J Surg Pathol. 2017:41:596-606.

21. Huang S-C, Zhang L, Sung Y-S, Chen C-L, Krausz T, Dickson BC, et al. Frequent FOS gene rearrangements in epithelioid hemangioma: a molecular study of 58 cases with morphologic reappraisal. Am J Surg Pathol. 2015;39:1313-21.

22. Trombetta D, Magnusson L, von Steyern FV, Hornick JL, Fletcher CDM, Mertens F. Translocation t (7;19)(q22;q13)-a recurrent chromosome aberration in pseudomyogenic hemangioendothelioma? Cancer Genet. 2011;204:211-5 Elsevier Inc.

23. Walther C, Tayebwa J, Lilljebjörn H, Magnusson L, Nilsson J, Von Steyern FV, et al. A novel SERPINE1-FOSB fusion gene results in transcriptional upregulation of FOSB in pseudomyogenic haemangioendothelioma. J Pathol. 2014;232:534-40.

24. Zhu G, Benayed R, Ho C, Mullaney K, Sukhadia P, Rios K, et al. Diagnosis of known sarcoma fusions and novel fusion partners by targeted RNA 
sequencing with identification of a recurrent ACTB-FOSB fusion in pseudomyogenic hemangioendothelioma. Mod Pathol. 2018. https://doi. org/10.1038/s41379-018-0175-7.

25. Requena L, Santonja C, Martinez-Amo JL, Saus C, Kutzner H. Cutaneous epithelioid Sarcomalike (Pseudomyogenic) Hemangioendothelioma: a littleknown low-grade cutaneous vascular neoplasm. JAMA Dermatol. 2013;149: 459-65.

26. Horan NA, Dimaio DJ. Pseudomyogenic Hemangioendothelioma. Cutis. 2017;100:E13-6.

27. Bryanton $M$, Makis W. Pseudomyogenic hemangioendothelioma mimicking multiple myeloma on 18F-FDG PET/CT, followed by spontaneous regression Clin Nucl Med. 2015;40:579-81.

28. Gabor KM, Sapi Z, Tiszlavicz LG, Fige A, Bereczki C, Bartyik K. Sirolimus therapy in the treatment of pseudomyogenic hemangioendothelioma. Pediatr Blood Cancer. 2018:65:4-7.

29. Ozeki M, Nozawa A, Kanda K, Hori T, Nagano A, Shimada A, et al. Everolimus for treatment of Pseudomyogenic Hemangioendothelioma. J Pediatr Hematol Oncol. 2017;39:e328-31.

30. Shah AR, Fernando M, Musson R, Kotnis N. An aggressive case of pseudomyogenic haemangioendothelioma of bone with pathological fracture and rapidly progressive pulmonary metastatic disease: case report and review of the literature. Skelet Radiol. 2015:44:1381-6.

31. van IJDGP, Sleijfer S, Gelderblom H, Eskens FALM, van LGJLH, Szuhai K, et al. Telatinib is an effective targeted therapy for Pseudomyogenic Hemangioendothelioma. Clin Cancer Res. 2018;24:2678-87.

\section{Publisher's Note}

Springer Nature remains neutral with regard to jurisdictional claims in published maps and institutional affiliations.

Ready to submit your research? Choose BMC and benefit from:

- fast, convenient online submission

- thorough peer review by experienced researchers in your field

- rapid publication on acceptance

- support for research data, including large and complex data types

- gold Open Access which fosters wider collaboration and increased citations

- maximum visibility for your research: over $100 \mathrm{M}$ website views per year

At BMC, research is always in progress.

Learn more biomedcentral.com/submissions 